\title{
GLAD!
}

Revue sur le langage, le genre, les sexualités

$05 \mid 2018$

Raconter les sexualités depuis la marge

\section{Come on. Discussion sur un nouveau mot qui émerge et qui va révolutionner notre manière de parler de sexe}

Come On. About a New Word Allowing to Speak Differently about Sex

\section{Bini Adamczak}

Traducteur : féministes dionysiennes

\section{(2) OpenEdition}

\section{Journals}

Édition électronique

URL : http://journals.openedition.org/glad/1401

ISSN : 2551-0819

Éditeur

Association GSL

Référence électronique

Bini Adamczak, «Come on. Discussion sur un nouveau mot qui émerge et qui va révolutionner notre manière de parler de sexe », GLAD! [En ligne], 05 | 2018, mis en ligne le 15 décembre 2018, consulté le 17 décembre 2020. URL : http://journals.openedition.org/glad/1401

Ce document a été généré automatiquement le 17 décembre 2020.

\section{cc) $(9)$}

La revue GLAD! est mise à disposition selon les termes de la Licence Creative Commons Attribution -

Pas d'Utilisation Commerciale - Pas de Modification 4.0 International. 


\title{
Come on. Discussion sur un nouveau mot qui émerge et qui va révolutionner notre manière de parler de sexe
}

\author{
Come On. About a New Word Allowing to Speak Differently about Sex
}

\section{Bini Adamczak}

Traduction : féministes dionysiennes

\section{RÉFÉRENCE}

Adamczak, Bini. 2016. « Come on. Diskussion Über ein neues Wort, das sich aufdrängt und unser Sprechen über Sex revolutionieren wird », analyse \& kritik 614. En ligne : www.akweb.de/ak_s/ak614/04.htm

1 Je propose un nouveau mot qui manque depuis trop longtemps. Il s'agit de circlusion. En langage plus vieillot on peut aussi dire circumclusion. La notion de circlusion s'oppose à celle de pénétration. Les deux mots décrivent à peu près le même processus matériel. Mais : d'une perspective opposée. Pénétration signifie introduire ou insérer. Circlusion signifie entourer, enrober ou enfiler par-dessus. Voilà. En utilisant le terme de circlusion, le rapport d'activité et de passivité est inversé. Pénétrer signifie: introduire quelque chose - un brin, une tige ou un bitoniau - dans quelque chose d'autre - un anneau ou un tuyau. Dans cette perspective, ce sont le brin, la tige ou le bitoniau qui sont considérés comme actifs. Circlure signifie : enfiler quelque chose - un anneau ou un tuyau - par-dessus quelque chose d'autre - un brin, une tige ou un bitoniau. Ici, ce sont l'anneau ou le tuyau qui sont actifs.

Le mot circlusion nous permet de parler autrement du sexe. Ce mot est nécessaire car cette triste fixette sur la pénétration domine toujours l'imaginaire hétéronormatif et comme si cela ne suffisait pas - domine aussi l'imaginaire queer. On peut l'observer 
dans le porno mainstream mais aussi dans le post-porno ou le porno BDSM. Presque sans exception, le gode et le pénis* servent de signe pratique de pouvoir.

Et cela, bizarrement, même chez les expertes du pouvoir. Les tops/dominantes de tout genre s'associent au gode, au pénis* et aux doigts érigés. Alors que les bottoms/subs sont plutôt associées à la bouche, au vagin*, à l'anus. Parfois, la vulve ou l'anus d'une personne domi semblent même être tabouisés, comme si les utiliser leur faisait perdre du pouvoir. Peut-être pas par rapport à la langue mais certainement par rapport au gode.

Bien évidemment, ce qui intéresse ici, ce n'est pas de savoir de quelles parties un corps est constitué, mais de quelles parties il se sert. Presque tous les corps disposent d'un anus, or quiconque s'en sert sexuellement - en rapport avec un gode, un pénis ou une main - est considéré comme bottom, comme soumis, comme passif. Presque toute personne peut s'offrir un gode-ceinture, mais quiconque s'en sert sexuellement sera habituellement considéré comme top, personne domi, actif-ve. What the Fuck ? Plutôt un anti-Fuck!

5 Il parait qu'il y ait des personnes qui pratiquent la baise génitale pendant quarante minutes - contractions du périnée, mouvements du bassin, sur une partenaire - et qui sont néanmoins de l'avis que ce sont elles qui ont été baisées. Et cela du simple fait qu'elles sont porteuses de vagin*/anus et que leur vis-à-vis sont porteur*euses de gode/pénis*. Le fantasme de la pénétration reste intact, même quand tous les faits indiquent le contraire. Frustrant.

6 Dans une société bourgeoise qui se fonde sur l'exploitation du travail des autres, le lien entre activité et pouvoir n'est de toute façon absolument pas plausible. Pas surprenant alors qu'on l'oublie tout de suite dès qu'on se met à sucer ou à lécher. Mais cela n'est pas notre sujet. Ce qui nous intéresse ici, c'est la connexion directe entre pénétration et pouvoir. Y en a marre - allez hop, à la poubelle! Inutile de rappeler toutes les associations que le discours de la pénétration dissémine dans son périmètre de signification. C'est souvent assez violent - il y a des perceuses et des trous, des verges, des épées, des gaines et autres objets qui semblent tout droit sortis d'affabulations.

7 Dans le langage des experts ainsi que dans celui du quotidien, la pénétration est en plus réduite aux pratiques qui sont accomplies avec un vagin*, un anus, un gode ou un pénis*. Doigts dans le cul, téton dans la bouche ne sont que rarement décrits comme du sexe pénétratif. Le mot circlusion n'a pas à partager cette limitation. Allègrement, il peut désigner l'activité d'une main autour d'un gode ou un vagin enfilé par-dessus un poing comme activité circluante. Mais ce n'est pas obligé. Comme la signification d'un signe émerge seulement dans son usage, la circlusion pourrait précisément prendre la place qu'avait précédemment la pénétration, et cela sans évoquer des images gênantes lors du sexe.

8 À titre d'exemple, on peut évoquer les cours de prévention de maladies sexuellement transmissibles. À personne ne viendrait l'idée de pousser une banane dans une capote fraichement déballée. C'est le monde à l'envers! Et puis, de toute façon, la circlusion fait déjà partie de notre expérience quotidienne. Pensons aux filets qui attrapent les poissons, le palais qui enrobe la nourriture, le casse-noix qui casse les noix, pensons à la main qui tient le levier ou la bouteille de bière.

9 En allemand, le terme penetrant est synonyme de aufdringlich (lourd, rentre-dedans). Le mot aufdringlich est formé de -dringen, qui renvoie à l'idée de pénétrer, et de auf- qui 
décrit l'action comme se déroulant par le dessus. Le terme penetrant devrait plutôt signifier eindringlich, formé avec ein-, qui renvoie à l'idée d'aller de l'extérieur vers l'intérieur. Prolétariennes de l'anus et de la bouche, du vagin et de la main : imposez vous - soyez aufdringlich! Parce qu'être aufdringlich signifie être circlusif! Dans ce sens, on pourrait dire: lorsqu'une vis est vissée dans un écrou, il y a pénétration; lorsque l'écrou est vissé sur la vis, il y a circlusion. Mais, en réalité, les deux procédés ont lieu simultanément.

10 La notion de circlusion nous permet ainsi de parler d'une expérience qui ne nous est pas nouvelle. Qui le souhaite peut continuer à utiliser la main ouverte, le vagin, l'anus ou la bouche pour être baisée. Ce qui est nouveau, c'est que nous pouvons aussi utiliser le doigt, le pénis*/gode ou le poing pour être baisée.e Évidemment, ce n'est pas comme si nous ne le faisions pas déjà. C'est juste qu'auparavant, il nous manquait un mot pour le dire.

11 Les pratiques langagières féministe sont souvent perçues comme compliquées. Or le mot circlusion est facile à apprendre et facile à appliquer. Je circlus, tu circlus, iel est circluse. Et surtout, il est beaucoup plus pratique que pénétration. Le mot pénétration comporte quatre syllabes, circlusion en a seulement trois. Ainsi son introduction est tout à fait dans l'intérêt de l'économie. Nous gagnons du temps précieux que nous pouvons ensuite investir dans la baise.

\section{RÉSUMÉS}

Ce texte propose un nouveau mot qui manque pour parler de sexualité : la circlusion, l'action d'entourer de manière serrée. Ce mot permet de construire un imaginaire queer affranchi de la domination hétéronormative.

This text suggests a new term to speak about sexuality that has been missing: circlusion. Circlusion is the action to push something onto something else. This term allows us to imagine a queer reality liberated from heteronormative domination

\section{INDEX}

Thèmes : Explorations

Keywords : circlusion, penetration, sex, power, feminist vocabulary

Mots-clés : circlusion, pénétration, sexe, pouvoir, vocabulaire féministe 


\section{AUTEURS}

\section{BINI ADAMCZAK}

Bini Adamczac travaille comme autrice, performeuse et artiste visuelle ; elle vit actuellement à Berlin. Elle a suivi des études en philosophie à Francfort. Son ouvrage Communism for Children a été publié en anglais aux MIT Press en 2017. 